\title{
Digital Manufacture of Shape Changing Components
}

\author{
Kai $\mathrm{Yu}^{1}$, Martin L. Dunn ${ }^{2 *}$, H. Jerry Qi ${ }^{1^{*}}$ \\ ${ }^{1}$ The George Woodruff School of Mechanical Engineering \\ Georgia Institute of Technology, Atlanta, GA 30332, USA \\ ${ }^{2}$ Digital Manufacturing and Design Centre (DManD) \\ Singapore University of Technology and Design, Singapore
}

*Corresponding author: martin_dunn@sutd.edu.sg; qih@me.gatech.edu 


\begin{abstract}
In this paper, we demonstrate the feasibility of controlling the shape changing sequence of shape memory polymers created from digital manufacturing by exploiting multi-shape memory effects. We create shape memory polymer components with precise architectures by 3D printing. After subjecting them to model-based thermomechanical programing steps, the components assume specified configurations in a precisely controlled shape changing sequence. The use of the 3D printing technique enables the digital manufacturing route with the advantages of easy implementation, large design freedom, and high printing resolution of shape memory polymer components. The results in this paper provide a method for precisely controlling the shape recovery profile and enabling the manufacture of devices with complicated geometries and unprecedented multifunctional performance.
\end{abstract}




\section{Introduction}

Among all of the shape memory materials, shape memory polymers (SMPs) have been the subject of extensive research in recent years due to desirable properties, such as high strain recovery ratio (up to 400\%) [1], low density, low cost, easy shape programming and controlling. Besides, SMPs can be chemically tuned to achieve biocompatibility and biodegradability. These advantages make SMPs attractive candidates for many applications such as microsystem actuation components, biomedical devices, aerospace deployable structures, and morphing structures [2-5].

Previous investigations on SMPs mainly focus on their dual-shape memory effect (SME), namely the continuous and spontaneous shape recovery from one temporary shape to the permanent shape after the material is exposed to an external stimulus, such as temperature [6-9], magnetic fields [10-13], light [14-17] and moisture [18, 19] etc. The functionality of SMPs can be further extended if one can effectively control the shape changing manner or sequence under a uniform stimulus magnitude. To achieve such controlled shape change, one strategy is to assign spatially independent compositions and hence independent thermomechanical properties, namely a functional gradient, within the polymer [20-22]. For example, Mather et al [22] successfully created functional SMPs where different sections of the material exhibit gradually increased glass transition temperature $\left(T_{g}\right)$ and consequently react to different temperatures independently. Other synthetic methods include UV polymerization with patterned photo-filters[23], photodegradation with a gradually removed mask[24], inter-diffusion of polymer bilayers [25] and co-extrusion with specially designed gradient distribution $[26,27]$ etc. The created SMP components, with properly assigned spatial variation of the thermomechanical 
properties, can respond rapidly to a thermal stimulus, and return to a specified configuration in a precisely controlled shape changing sequence.

Another strategy to control shape changing of SMPs involves exploring additional temporary shapes in a shape memory cycle to achieve multi-SME. One approach is to integrate discrete reversible transitions into a single SMP [28-32] system or structure. For example, Bellin and co-workers [33] combined two different polymer segments into a macroscopically homogeneous network. These two segments, which are capable of glass transition and melt transition respectively, can be activated at different recovery temperatures and hence enable the triple-SME in the SMP network. Xie et al. [32] introduced a different method of achieving the triple-SME using a macroscopic bilayer crosslinked polymer structure with two well separated phase transitions. Recently, Luo et al. [31] reported SMP composites with triple-SME, where an epoxy SMP works as the matrix and provides one glass transition, while the non-woven PCL (poly( $\varepsilon$-caprolactone)) microfibers works as fillers and provides the other melt transition.

In addition to tailor different transitions, the multi-SME can be achieved by properly designing the programing and recovery conditions for polymers with a broad range of transition temperature. As a notable example, Xie et al [34-37] demonstrated that by successively programming and applying step-heating methods during the free recovery, the material (a Nafion membrane) reached different temporary shapes at different recovery temperatures. Previously, by using a multi-branched thermoviscoelastic model [36], we studied the energy release mechanism during such multi-SME. The results indicate that the energy state of each recovered temporary shape is equivalent to that of corresponding programming shape. This means the shape recovery manner and sequence 
can be easily designed by successively programming the material into the same shapes, which is considered to be the advantage of this method in achieving sequential shape changings in the SMPs. This work also indicates that multi-SMEs can be observed in any polymer system that exhibits a broad glass transition region.

In this paper, we combine the multi-SMEs with the 3D printing technique [38-44] to create $3 \mathrm{D}$ printed components and structures with both spontaneous and controlled shape changing properties. The digital manufacturing method involves direct 3D printing from a CAD file that specifies the configuration details of SMPs and their components. This method enables the creation of solids with complex geometries and material distributions. The multi-SME is realized in the printed SMP components by following Xie's approach [34-37], where the material is successively programed into different temporary shapes at different programing temperatures. A multi-branched constitutive model is applied to assist the selection of programing and recovery conditions to achieve distinguishable shape changes. In addition to 1D uniaxial shape recovery, we demonstrate the characteristics of multi-SMEs that involve bending deformation in different directions, as well as that in SMP components. As will be shown, this paper uses only one single printed material to achieve multiple shape change, which is different from our previous work $[45,46]$ where multiple materials were used. The results in this paper provide a potential digital manufacturing and operating route for precisely controlling the shape recovery profile and enabling the fabrication of devices with multifunctional performance. Although we limit ourselves to multi-SME in relatively simple geometries in this paper, more complicated temporary shapes can be explored and utilized in the 3D printed SMPs with broader glass transitions. 


\section{Experiments}

\subsection{Material and manufacturing}

All the SMPs and their components used in this study were manufactured by the polyjet 3D printing method, where droplets of inks are selectively deposited onto a build platform. A commercial 3D multimaterial polymer printer (Objet Connex 260, Stratasys, Edina, MN, USA) was used for the fabrication, and the associated inks (epoxy based photo-curable resin) were used without modification.

During the operation of the printer, after a layer of droplets is deposited at $\sim 70{ }^{\circ} \mathrm{C}$, a roller evens out the horizontal surface of the layer, followed by UV light irradiation passing over the printed layer to cure the polymer. A more detailed description of the manufacturing process can be found in Stiltner et al.[47]. As several inkjet printheads with separate material sources can be installed into the printing block, multiple materials can be deposited in a single layer, enabling the creation of digital materials. Since SMPs with broad glass transition range typically exhibit clear multi-SME [34], we select the digital material Gray 60 in our 3D printer's material library to fabricate SMP components. Gray 60 has the glass transition range $\sim 40^{\circ} \mathrm{C}$ (see Section 3.1 for detailed characterizations). Besides, the glass transition temperature of Gray 60 is above the room temperature, so the programed temporary shape can be effectively fixed at the room temperature.

\subsection{Dynamic mechanical analysis}

Dynamic mechanical analysis (DMA) was used to characterize the thermomechanical properties of the printed SMP. The sample dimension was $15 \mathrm{~mm} \times 2 \mathrm{~mm} \times 0.8 \mathrm{~mm}$ and the tests were performed on a DMA machine (Model Q800, TA Instruments, New Castle, DE, USA) in the uniaxial tension mode. During the tests, the SMP was first heated to 100 
${ }^{\circ} \mathrm{C}$ and stabilized for 20 minutes to reach the thermal equilibrium, and then a preload of 1 KPa was applied. During the DMA experiment, the strain was oscillated at a frequency of $1 \mathrm{~Hz}$ with a peak-to-peak amplitude of $0.1 \%$ while the temperature was decreased from $100{ }^{\circ} \mathrm{C}$ to $0{ }^{\circ} \mathrm{C}$ at a rate of $2{ }^{\circ} \mathrm{C} / \mathrm{min}$.

\subsection{Shape memory behavior characterization}

The 1D multi-SME of the printed SMP was characterized on the DMA machine. For a triple-SME, there were two programming steps. In the first one, the SMP was stretched by a constant stress at the first programming temperature $\left(T_{d l}\right)$. This stress was maintained while the temperature was lowered to the second programming temperature $\left(T_{d 2}\right)$ at the rate of $2^{\circ} \mathrm{C} \min ^{-1}$. When the temperature reached $T_{d 2}$, the SMP was equilibrated for $5 \mathrm{~min}$. Afterwards, the external force was removed, leading to a partial recovery. After 5 min of equilibrium, the remaining (fixed) strain corresponded to the first temporary shape. In the second programming step, the material was stretched by a constant stress at $T_{d 2}$. The temperature was then lowered to $T_{L}\left(20^{\circ} \mathrm{C}\right)$ at $2{ }^{\circ} \mathrm{C} \min ^{-1}$ and the external load was maintained for another 5 min before its removal. This marked the end of the programming process. During the recovery, the SMP was heated under a stress free condition. In the first recovery step, the temperature was ramped to the first recovery temperature $T_{r l}$ with a heating rate of $2{ }^{\circ} \mathrm{C} \mathrm{min}^{-1}$ and then held constant for $30 \mathrm{~min}$. Further heating of the SMP to $T_{r 2}$, followed by isothermal holding, led to the second recovery.

For the multi-SME in the SMP components studied in this paper, the structures were manually deformed in the programming step in a thermal chamber (Thermcraft, Model LBO, Winston Salem, NC, USA). The temperature was controlled by a Eurotherm 
controller (Model Euro 2404, Chesterfield, VA, USA) where a built-in electrical heater with a fan provided the heat. During the operation, all the SMP components were horizontally placed on a glossy glass slide to minimize the resistance from the gravity and friction to the shape recovery.

\section{Results and discussions}

\subsection{Glass transition behavior and model parameters identification}

Fig. 1 shows the glass transition behavior of the printed SMP (Gray 60), where the solid lines denote the storage modulus and $\tan \delta$ within the temperature variation range. It is seen the storage modulus decreases with temperature. The temperature corresponding to the peak of the $\tan \delta$ curve is taken to be the glass transition temperature $T_{g}\left(\sim 64{ }^{\circ} \mathrm{C}\right)$. Fig. 1 also shows the glass transition range (from $\sim 40{ }^{\circ} \mathrm{C}$ to $\sim 80{ }^{\circ} \mathrm{C}$ ) of the printed SMP materials; based on this, we select a temperature range from $T_{L}=20{ }^{\circ} \mathrm{C}$ (shape fixing temperature) to $T_{d l}=80{ }^{\circ} \mathrm{C}$ (first programming temperature) for examining the multiSME.

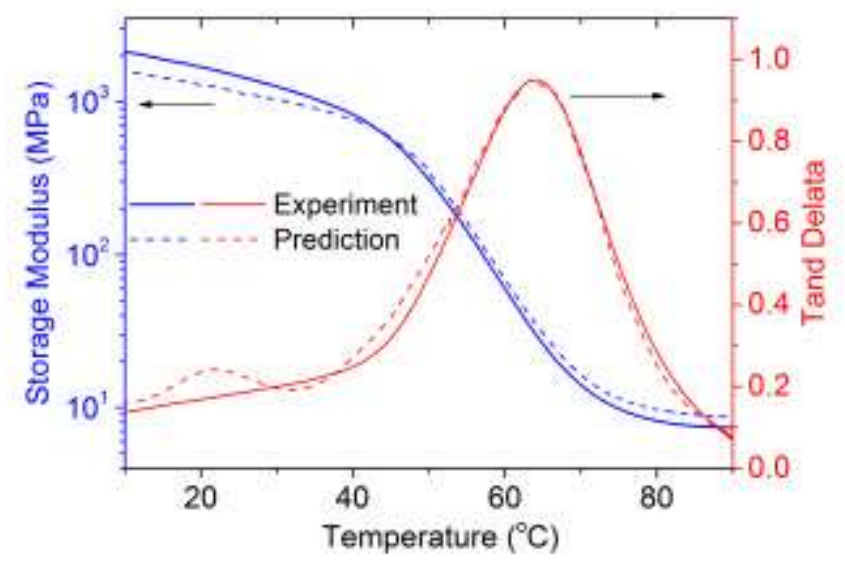

Figure 1. Glass transition of the printed SMP. Solid lines denote experimental data and dash lines denote model predictions by using the multi-branched model 
In our study, a multi-branched constitutive model was applied to capture the glass transition behavior of the created SMP material, and subsequently to predict its shape memory behavior. A description of the multi-branch model can be found in the Supplementary Material. The storage modulus and $\tan \delta$ curves are used to determine the model parameters. The basic procedure is to use nonlinear regression method to fit the experimental curves. A model parameter identification method of the multi-branch model can be found in our previous study $[48,49]$. As shown in Fig. 1, the identified parameters could enable the multi-branch model to successfully capture the experimental storage modulus and $\tan \delta$ curves within the entire testing temperature range $\left(10^{\circ} \mathrm{C}-90^{\circ} \mathrm{C}\right)$.

\section{2 $1 D$ triple-shape memory effect}

We first tested the triple-SME in the printed SMP under the uniaxial deformation. The shape fixity $R_{f}$ is calculated by the spring back deformation $\Delta e$ during the unloading step:

$$
R_{f}=\left(e_{\max }-\Delta e\right) / e_{\max },
$$

where $e_{\max }$ is the strain before unloading. The shape recovery ratio is based on the strain evolution $e(t)$ in the recovery step and calculated as:

$$
R_{r}=1-e(t) /\left(e_{\max }-\Delta e\right)
$$

The temperature and strain evolution during the shape memory cycle are shown in Fig. 2. During the first programming step, the material was stretched by a stress of 0.67 $\mathrm{MPa}$ at the first programming temperature $T_{d l}=80^{\circ} \mathrm{C}$. The resulted engineering strain was $9 \%$. Afterwards, the temperature was lowered to $T_{d 2}=50{ }^{\circ} \mathrm{C}$ at the rate of $2{ }^{\circ} \mathrm{C} \min ^{-1}$, followed by sample unloading. After being stabilized for $5 \mathrm{~min}$ in the stress-free state, the programing strain in the SMP was decreased to $7.8 \%$ due to partial recovery. In the second programming step, the material was stretched at $T_{d 2}=50^{\circ} \mathrm{C}$ by a stress of $1.2 \mathrm{MPa}$, 
which led to the programming strain further increasing to $17.8 \%$. The temperature was then lowered to $T_{L}=20{ }^{\circ} \mathrm{C}$ at $2{ }^{\circ} \mathrm{C} \mathrm{min}{ }^{-1}$ and the external load was maintained for another 5 min before it was removed. This marked the end of the programming process, and the shape fixity was $97.8 \%$.

During the recovery step, the SMP was heated under a stress-free condition to induce the strain recovery. In the first recovery step, the temperature was ramped to the first recovery temperature $T_{r l}=50{ }^{\circ} \mathrm{C}$ with a heating rate of $2{ }^{\circ} \mathrm{C} \mathrm{min}{ }^{-1}$ and then held constant for $30 \mathrm{~min}$. The shape recovery ratio at this moment was $49 \%$. Further heating of the SMP to $T_{r 2}=80{ }^{\circ} \mathrm{C}$, followed by isothermal holding, led to the second recovery. The final shape recovery ratio was $100 \%$. In addition to the experimental testing, Fig. 2 also showed that the multi-branched model with the determined model parameters captured the overall strain evolution during the entire shape memory cycle.

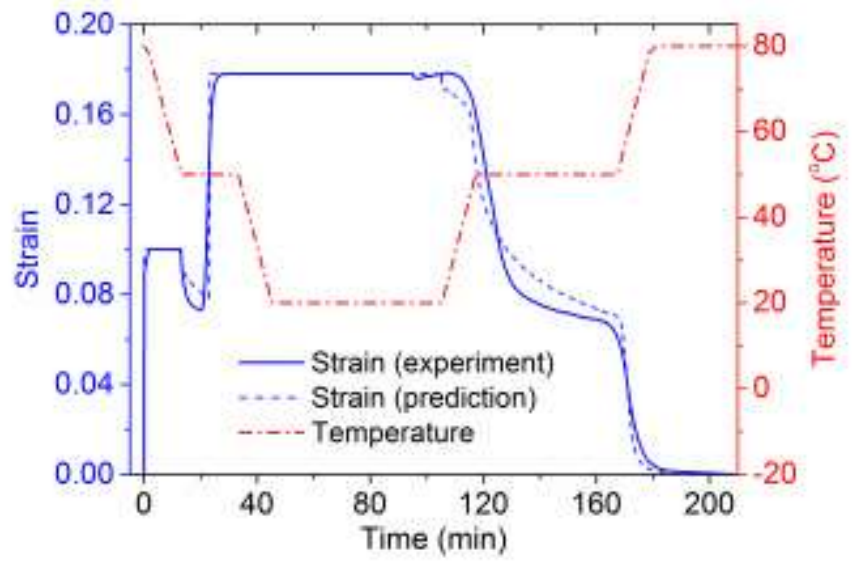

Figure 2. Triple-shape memory effect of the printed SMP. Solid lines denote experimental data and dash lines denote model prediction using the multi-branched model.

Since the shape memory performance strongly depends on the selected thermaltemporal conditions in a shape memory cycle $[50,51]$, parametric studies were performed both experimentally and theoretically to help determine the programming and recovery 
conditions for achieving a clear triple-SME. The parametric variables were chosen to be the second programming temperature $T_{d 2}$ and first recovery temperature $T_{r l}$, while the rest parameters were set to be constant. Fig. 3 summarizes the shape recovery ratio at the end of the first recovery step in each case. In addition to the consistence between experimental results and model predictions, the figure reveals that when the second programming temperature $T_{d 2}$ is between $30^{\circ} \mathrm{C}$ and $55^{\circ} \mathrm{C}$ for the case of $T_{r 1}=50{ }^{\circ} \mathrm{C}$, or $T_{d 2}$ is between $30^{\circ} \mathrm{C}$ and $45^{\circ} \mathrm{C}$ for the case of $T_{r l}=40{ }^{\circ} \mathrm{C}$, the first recovery step will recovery $\sim 50 \%-\sim 60 \%$ of the shape, thus will provide two clear shapes. For the programming temperature, in our previous studies $[36,52]$, we found the programming temperature will affect energy storage and thus will affect the recovery of SMPs. In the first programming step of triple-SME with a higher programming temperature $\left(T_{d 2}=80^{\circ} \mathrm{C}\right)$, most dashpots will develop almost the same viscous strain due to their small relaxation times, while in the second programming step, the viscous strains depend on the distribution of relaxation times in the dashpot. In the nonequilibrium branches with larger relaxation times, significant elastic energy will be developed, which provides additional driving force for the shape recovery and increases viscous strain release rate in the first recovery step [52]. In this manner, decreasing the second programming temperature $T_{d 2}$ will increase the first recovery ratio with the same recovery condition. However, a further decrease of $T_{d 2}$ will also lead to a saturated state [52] where the first shape recovery ratio is independent of $T_{d 2}$. In the following discussions, we focus on demonstrating a clear multi-SME based on the theoretical results shown in Fig. 3. 


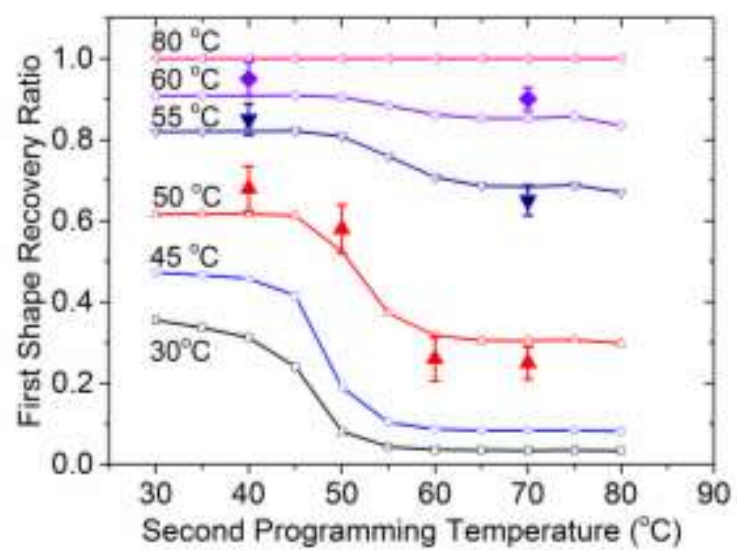

Figure 3. The shape recovery ratio at the end of the first recovery step as a function of $T_{d 2}$ and $T_{r 1}$. Temperature marked in the figure is the first recovery temperature $T_{r}$. Isolated symbols with error bars indicate the experimental results. Three identical shape memory cycles were tested for each case.

\subsection{Triple-shape shape memory effect with bending deformation}

Here, we demonstrate the triple-shape SME with bending deformation, where a printed SMP strip, with the dimension of $76.6 \mathrm{~mm} \times 2.5 \mathrm{~mm} \times 1 \mathrm{~mm}$, was programmed and heated for recovery in the oven. Fig.4 shows snapshots during the shape memory cycle. The first programming step involves rolling the sample clockwise around a cylinder mold (diameter $=24.4 \mathrm{~mm}$ ) for $\sim 360^{\circ}$ at $T_{d l}=80^{\circ} \mathrm{C}$. After the temperature was lowered to $T_{d 2}=50^{\circ} \mathrm{C}$, the external load was removed and the SMP sample reaches the first programming shape. This finished the first programing step. Subsequently, at the same temperature $T_{d 2}=50^{\circ} \mathrm{C}$, the sample was bent counter-clockwise around the same mold for $\sim 360^{\circ}$. The second programing shape was fixed after unloading at $T_{L}=20^{\circ} \mathrm{C}$.

We adopt the bending curvature to quantify the shape changing during the SM cycle. By using the curvature, one can easily inspect the strain distribution inside the SMP sample. Due to the uniform bending angle, it is reasonable to assume a constant curvature for each state as shown in Fig. 4, which is taken to be the inverse of corresponding curve radius. Besides, we also assume a counter-clockwise bending corresponding to a negative 
curvature. By using these definition and assumptions, the target bending curvature during the two programing steps are calculated as $8.2 \times 10^{-2} \mathrm{~mm}^{-1}$ and $-8.2 \times 10^{-2} \mathrm{~mm}^{-1}$ respectively, and the curvature for the two temporary shapes are $7.1 \times 10^{-2} \mathrm{~mm}^{-1}$ and -7.9 $\times 10^{-2} \mathrm{~mm}^{-1}$. In analogy to the definition in Eq. 1, the shape fixities for these two programing steps are $86.6 \%$ and $96.3 \%$ respectively.

During the first recovery step, the temperature was firstly ramped to $T_{r l}=50^{\circ} \mathrm{C}$ at $2^{\circ} \mathrm{C} / \mathrm{min}$. Since the deformation developed in the second programming step would be recovered first, the SMP sample gradually returned towards the first programmed shape within the first 30min of heating. Further increasing the recovery temperature to $T_{r 2}=80^{\circ} \mathrm{C}$ led to a full shape recovery within $10 \mathrm{~min}$. Although Fig. 2 shows that in a uniaxial shape memory cycle, the first recovered shape of SMP is close to the first temporary shape, the experimental result in Fig. 4 shows a difference between these two shapes when the SMP sample is subjected to bending deformation. As shown in the figure, the bending curvature is decreased by $15 \times 10^{-2} \mathrm{~mm}^{-1}$ (from $7.1 \times 10^{-2} \mathrm{~mm}^{-1}$ to -7.9 $\times 10^{-2} \mathrm{~mm}^{-1}$ ) in the second programing step, while it is increased by $13 \times 10^{-2} \mathrm{~mm}^{-1}$ (from $7.9 \times 10^{-2} \mathrm{~mm}^{-1}$ to $5.1 \times 10^{-2} \mathrm{~mm}^{-1}$ ) during the first recovery step, which indicates an $87 \%$ recovery with respect to the first temporary shape. 


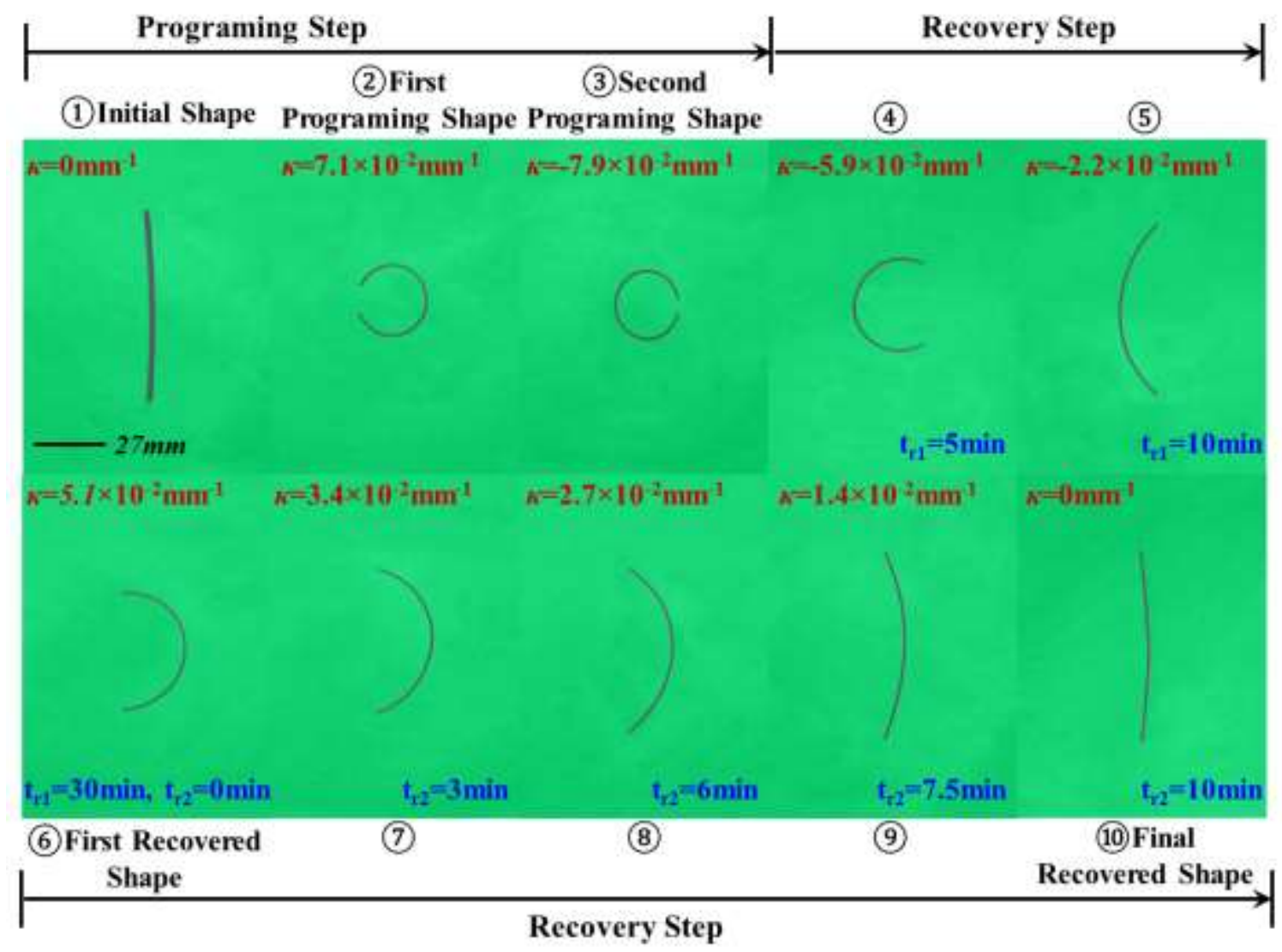

Figure 4. Snapshots during the triple-shape memory cycle. $t_{r l}$ denotes the heating time in the first recovery step, and $t_{r 2}$ denotes the heating time in the second recovery step

It is interesting to see if we can improve the bending recovery performance by slightly adjusting the prorgamming and recovery temperautres. In the following, we use different $T_{d 2}$ and $T_{r l}$ respectively to examine their influence. Fig. 5 sumarizes the first recovered shape after $30 \mathrm{~min}$ of heating in each case, where the first programmed shape is also shown in the figure for comparasion. It can be seen that if the $T_{d 2}$ is higher than $T_{r 1}$ (first row in the right group of Fig.5), the curvature change in the first recovery step is less than that in the second programing step. For example, if $T_{d 2}=60^{\circ} \mathrm{C}$, which is $10^{\circ} \mathrm{C}$ higher than $T_{r l}$, the recovered curvature in the first recovery step is only $\sim 12.7 \%$ with respect to the second temporary shape. On the other hand, when the $T_{d 2}$ is lower than $T_{r 1}$, some portion of programming curvature in the first programing step will be recovered. In 
this manner, the first recovered shape is closer to the permanent shape of the SMP. These trends are consistent with the predictions demonstrated in Fig. 3.

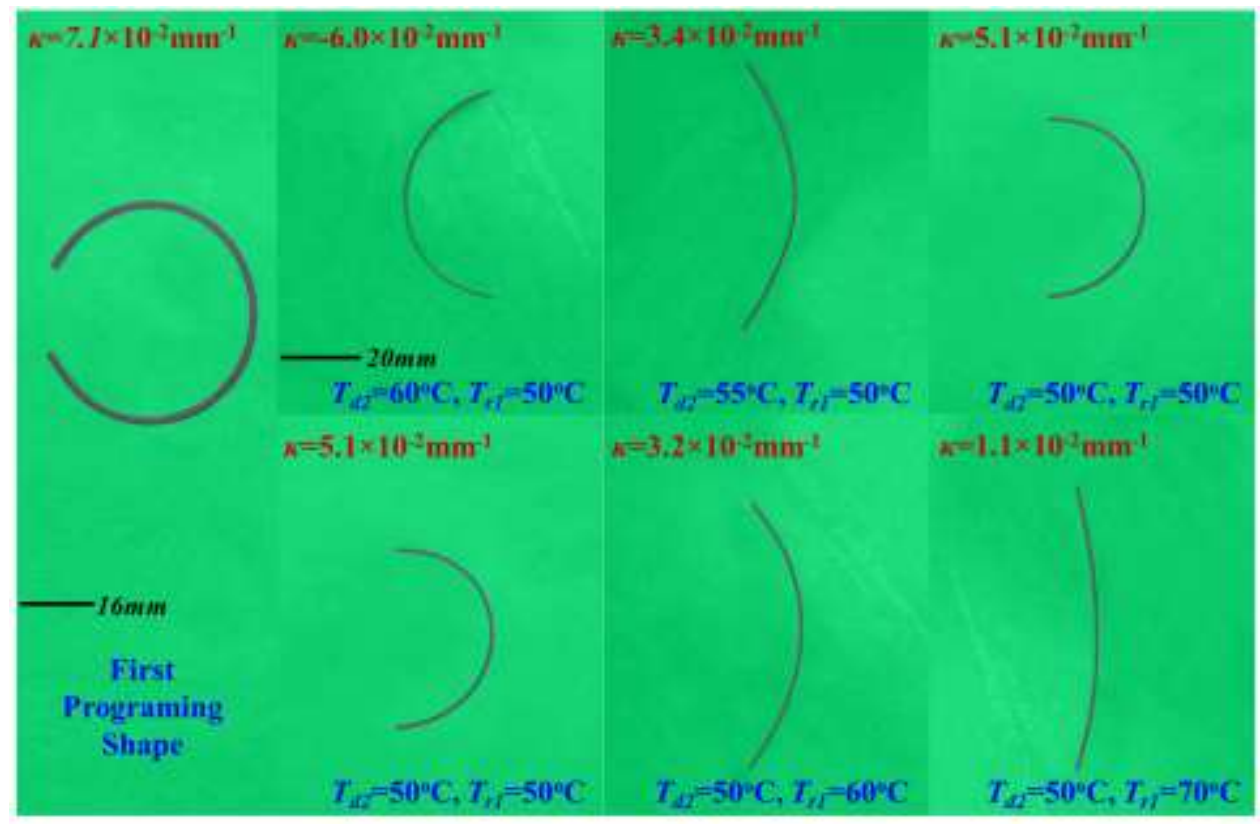

Figure 5. Comparasion between the first programming shape and the first recovered shape when different $T_{d 2}$ and $T_{r 1}$ are applied. The first programming temperature is $T_{d 2}=80^{\circ} \mathrm{C}$.

Experimental investigations in Fig. 4 and Fig. 5 show that equalizing $T_{d 2}$ and $T_{r l}$ at 50 ${ }^{\circ} \mathrm{C}$ leads to best bending triple-SME. To examine if the difference between the first temporary shape and the first recovered shape (as seen in Fig. 4) is resulted from the different deformation manner or some other material behaviors beyond consideration, we extend the aforementioned 1D multi-branched model into 3D with the same model parameters and constitutive relations. Finite element method (FEM) simulations were then conducted to investigate the bending shape recovery of SMPs. A detailed description of the model and FEM simulation is presented in the supplementary material.

Fig. 6 shows the simulation results as well as the comparison with the experimental results. As shown in Fig. 6a, with the same thermal temporal conditions, the simulated bending curvature is firstly decreased by $15.7 \times 10^{-2} \mathrm{~mm}^{-1}$ (from $7.5 \times 10^{-2} \mathrm{~mm}^{-1}$ to -8.2 
$\times 10^{-2} \mathrm{~mm}^{-1}$ ) in the second programing step, and then increased by $15.4 \times 10^{-2} \mathrm{~mm}^{-1}$ (from $-8.2 \times 10^{-2} \mathrm{~mm}^{-1}$ to $7.2 \times 10^{-2} \mathrm{~mm}^{-1}$ ) during the first recovery step, which is corresponding to a $\sim 98 \%$ recovery. The simulation result indicates that if the SMPs possess isotropic thermomechanical properties, the bending recovery ratio should be quantitatively consistent with the uniaxial recovery ratio (see Fig. 2) due to the linear relation between bending curvature and uniaxial strains along the bending layers. We conjecture that the experimentally observed difference between the first temporary shape and the first recovered shape might be due to the anisotropic nature of printed SMP strip. In addition, interfacial areas between printing layers may exhibit distinct shear modulus and shape recovery properties. These will be studies in the future. However, since difference between the two bending shapes is not significant, we assume the first temporary shape could be basically recovered in the first recovery step.
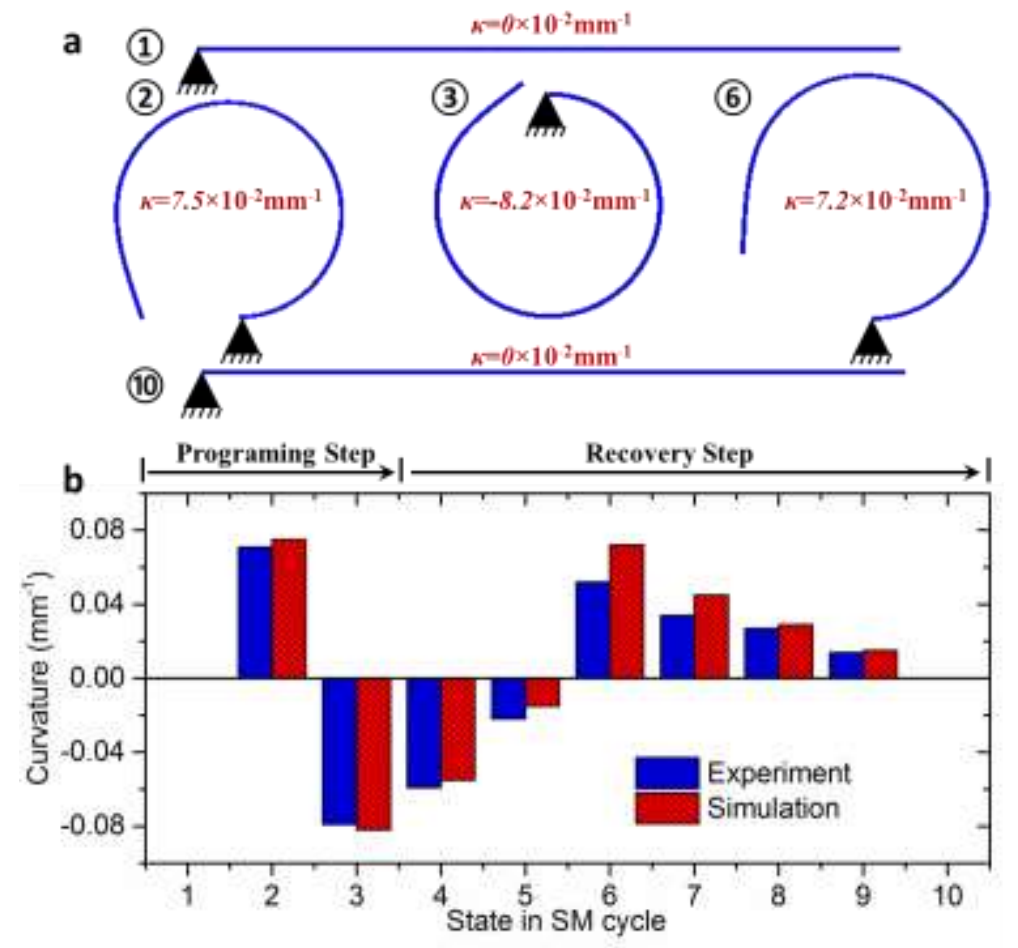

Figure 6. Finite element method simulation on the triple-SME with bending deformation (a) as awell as its comparasion with experimental results (b). Note: in Fig. 6a, (1),2, (3),6 
and (10)represent the initial state, first temporary shape, second temporary shape, first recovered shape and final state respectively. In Fig. 6b, states 1-10 in the shape memory cycle are corresponding to the ten snapshots of Fig 4.

\subsection{Triple-shape memory effect in SMP components}

In additon to the simple bending, we further explore how the polymer triple-SME can be achieved in the printed SMP components where the interplay between the triple-SME and the material geometry can be exploited to realize components that can assume prescribed configurations to performing desired functions. Fig. 7a shows the triple-SME in a printed SMP square, with a dimension of $64 \mathrm{~mm} \times 64 \mathrm{~mm}$ and a thickness of $1 \mathrm{~mm}$. Here, the $T_{d 2}$ and $T_{r 1}$ were all set to be $50^{\circ} \mathrm{C}$. The opposite sides were successively bent inward during the two programing steps. The corresponding curvatures are marked in the figure, where $\kappa_{1}$ denotes the curvature of the two horizontal sides, and $\kappa_{2}$ denotes the curvature of the two vertical sides. The curvature in each state of the shape memory cycle is summarized in Fig. $7 \mathrm{~b}$.

Overall, the shape changing sequence of the printed SMP square follows a tripleshape memory cycle, where the first programming shape is firstly recovered during the first recovery step. Close examination of each side reveals distinct shape changing mechanism. For horizontal sides, curvature is only developed in the second programming step $\left(T_{d 2}=50{ }^{\circ} \mathrm{C}\right)$, which is totally released in the first recovered step $\left(T_{r l}=50{ }^{\circ} \mathrm{C}\right)$ and the sides remain stable in the second recovery step. Such a shape recovery manner indeed represents the temperature memory effect $[34,35,53]$, namely the programing strain developed in a lower programming temperature will be firstly released in a lower recovery temperature. For the vertical sides, the shape changing manner follows the triple-shape memory cycle as demonstrated in section 3.4 , where the bending curvature is 
firstly decreased by $7.81 \times 10^{-2} \mathrm{~mm}^{-1}$ (from $8.1 \times 10^{-2} \mathrm{~mm}^{-1}$ to $0.29 \times 10^{-2} \mathrm{~mm}^{-1}$ ) in the second programing step, and then increased by $6.71 \times 10^{-2} \mathrm{~mm}^{-1}$ (from $0.29 \times 10^{-2} \mathrm{~mm}^{-1}$ to $7.0 \times 10^{-2} \mathrm{~mm}^{-1}$ ) during the first recovery step, which is corresponding to a $\sim 85 \%$ recovery. The interplay among all the four square sides leads to the controlled shape changing sequence of the box, namely the successive deployment in the horizontal and vertical directions during the recovery steps. 


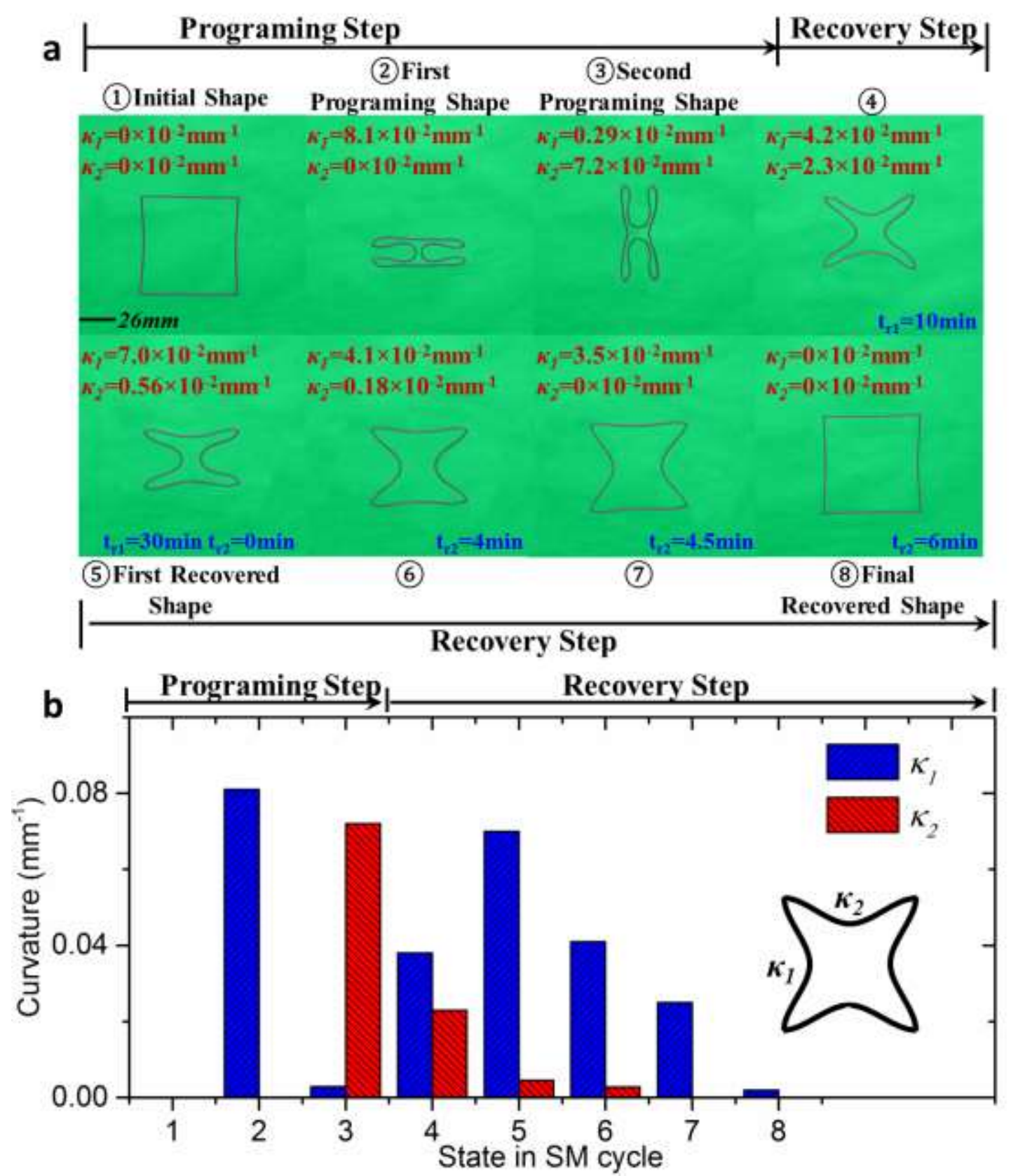

Figure 7. Triple-SME in the printed SMP box. (a) Snapshots during the shape memory cycle, where $\kappa_{1}$ denotes the curvature of the two horizontal sides, and $\kappa_{2}$ denotes the curvature of the two vertical sides. (b) Summary of the curvatures during the shape memory cycle. States 1-8 in the shape memory cycle are corresponding to the eight snapshots of Fig 7a.

The triple-SME is also realized in a printed SMP truss structure, where hinges are designed at the connections to enable local rotation (shown in Fig. 8). The total length of the structure before programming is $261 \mathrm{~mm}$ and the width is $67 \mathrm{~mm}$. During the first 
programing step, the SMP truss was compressed by $71.3 \%$ in the vertical direction at $T_{d 1}=80^{\circ} \mathrm{C}$. After unloading at $T_{d 2}=50^{\circ} \mathrm{C}$, the truss structure was stretched by $113 \%$ compared with the first programing shape. This shape was fixed after unloading at $T_{L}=20^{\circ} \mathrm{C}$. When the recovery temperature was successively ramped to $T_{r l}=50^{\circ} \mathrm{C}$ and $T_{r 2}=80^{\circ} \mathrm{C}$, the structure first shrunk towards the first programmed shape and then extended in the vertical direction until it fully recovered. Such sequential shape changing represents a synergistic triple-SME in each bended hinge.

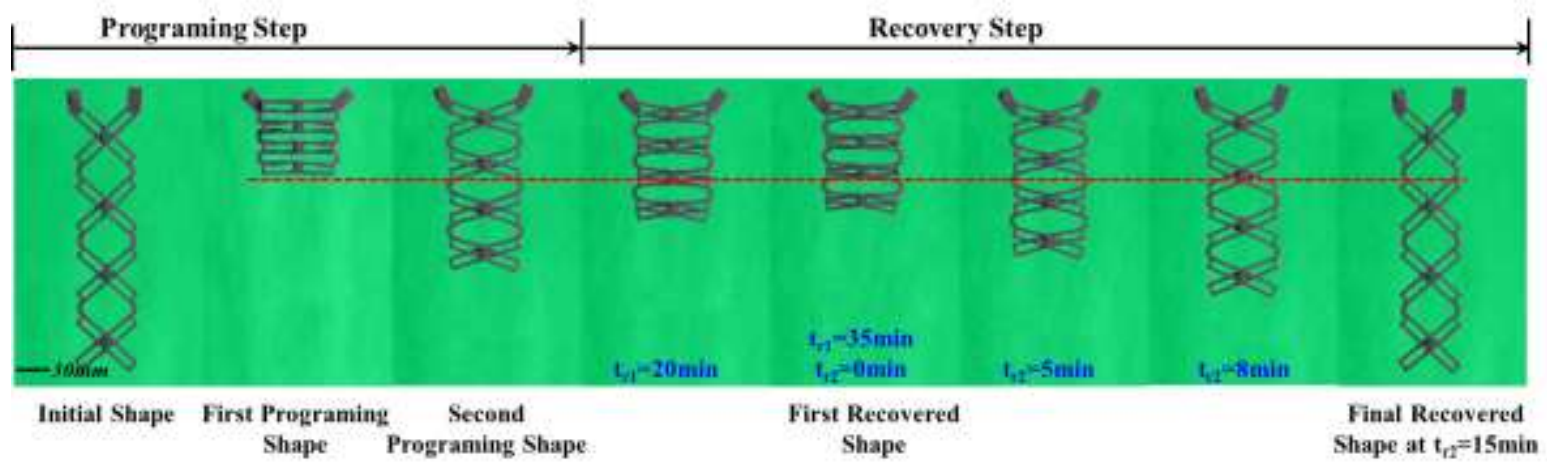

Figure 8. Triple-SME in the printed SMP truss structure.

\section{Conclusion}

In this paper, we experimentally demonstrated the feasibility of controlling the shape changing sequence of shape memory polymers created from digital manufacturing by exploiting multi-shape memory effects. For the created shape memory polymer components, the programmed temporary shape at a given programing temperature could be recovered in the recovery step when heating the material to the same temperature. Although some difference was observed between the first programmed shape and the first recovered shape, the overall shape change followed the programmed ones. Such an easy and straightforward routine provides considerable freedom in designing and controlling the shape change and sequence of functional shape memory polymers, and makes 
possible the implementation of the polymer shape memory effect in a wide array of objects and applications yet to be realized, such as structures involving complex deformation permutations, and versatile shape memory polymer solids able to self-adjust and self-reinforce to cope uniquely with different environmental conditions etc. By using the $3 \mathrm{D}$ printing technique, the shape memory polymer structures with highly controlled sequential shape changing capabilities could be directly created with large design freedom and high resolution of material properties distribution. Besides, the manufacturing route can be easily implemented and potential for immediate engineering applications for low-cost, rapid production.

\section{Acknowledgements}

We gratefully acknowledge the support of an AFOSR grant (FA9550-13-1-0088; Dr. B.-L. "Les" Lee, Program Manager). HJQ acknowledge the support of the NSF award (CMMI-1462894, CMMI-1462895, and EFRI-1435452). MLD acknowledges support from the SUTD Digital Manufacturing and Design Centre (DManD), supported by the Singapore National Research Foundation.

\section{References}

1. Wei, Z.G., R. Sandstrom, and S. Miyazaki, Shape-memory materials and hybrid composites for smart systems - Part I Shape-memory materials. Journal of Materials Science, 1998. 33(15): p. 3743-3762.

2. Yakacki, C.M., et al., Unconstrained recovery characterization of shape-memory polymer networks for cardiovascular applications. Biomaterials, 2007. 28(14): p. 2255-2263.

3. Lendlein, A. and S. Kelch, Shape-memory polymers as stimuli-sensitive implant materials. Clinical Hemorheology and Microcirculation, 2005. 32(2): p. 105-116.

4. Liu, Y.P., et al., Thermomechanics of shape memory polymer nanocomposites. Mechanics of Materials, 2004. 36(10): p. 929-940.

5. Francis, W.H., et al. Elastic Memory Composite Microbuckling Mechanics: Closed-Form Model with Empirical Correlation. in 48th AIAA/ASME/ASCE/AHS/ASC Structures, Structural Dynamics, and Materials Conference. 2007. Honolulu, Hawaii.

6. Lendlein, A. and S. Kelch, Shape-memory polymers. Angew Chem Int Ed Engl, 2002. 41(12): p. 2035-57. 
7. Liu, C., H. Qin, and P.T. Mather, Review of progress in shape-memory polymers. Journal of Materials Chemistry, 2007. 17(16): p. 1543-1558.

8. Mather, P.T., X.F. Luo, and I.A. Rousseau, Shape Memory Polymer Research. Annual Review of Materials Research, 2009. 39: p. 445-471.

9. Ge, Q., et al., Prediction of temperature-dependent free recovery behaviors of amorphous shape memory polymers. Soft Matter, 2012. 8(43): p. 11098-11105.

10. Mohr, R., et al., Initiation of shape-memory effect by inductive heating of magnetic nanoparticles in thermoplastic polymers. Proceedings of the National Academy of Sciences of the United States of America, 2006. 103(10): p. 3540-3545.

11. Schmidt, A.M., Electromagnetic activation of shape memory polymer networks containing magnetic nanoparticles. Macromolecular Rapid Communications, 2006. 27(14): p. 1168-1172.

12. $\mathrm{Yu}, \mathrm{K}$. , et al., Design considerations for shape memory polymer composites with magnetic particles. Journal of Composite Materials, 2013. 47(1): p. 51-63.

13. He, Z.W., et al., Remote Controlled Multishape Polymer Nanocomposites with Selective Radiofrequency Actuations. Advanced Materials, 2011. 23(28): p. 3192-3196.

14. Jiang, H.Y., S. Kelch, and A. Lendlein, Polymers move in response to light. Advanced Materials, 2006. 18(11): p. 1471-1475.

15. Lendlein, A., et al., Light-induced shape-memory polymers. Nature, 2005. 434(7035): p. 879-882.

16. Scott, T.F., R.B. Draughon, and C.N. Bowman, Actuation in crosslinked polymers via photoinduced stress relaxation. Advanced Materials, 2006. 18(16): p. 2128-+.

17. Scott, T.F., et al., Photoinduced plasticity in cross-linked polymers. Science, 2005. 308(5728): p. 1615-1617.

18. Huang, W.M., et al., Water-driven programmable polyurethane shape memory polymer: Demonstration and mechanism. Applied Physics Letters, 2005. 86(11): p. -.

19. Jung, Y.C., H.H. So, and J.W. Cho, Water-responsive shape memory polyurethane block copolymer modified with polyhedral oligomeric silsesquioxane. Journal of Macromolecular Science Part B-Physics, 2006. 45(4): p. 453-461.

20. Yang, B., et al., Effects of moisture on the glass transition temperature of polyurethane shape memory polymer filled with nano-carbon powder. European Polymer Journal 2005. 41(5): p. 1123-1128.

21. Yang, B., et al., Effects of moisture on the thermomechanical properties of a polyurethane shape memory polymer. Polymer, 2006. 47(4): p. 1348-1356.

22. DiOrio, A.M., et al., A functionally graded shape memory polymer. Soft Matter, 2011. 7(1): p. 68-74.

23. Wong, J.Y., et al., Directed movement of vascular smooth muscle cells on gradientcompliant hydrogels. Langmuir, 2003. 19(5): p. 1908-1913.

24. Yao, X.F., D.L. Liu, and H.Y. Yeh, Mechanical properties and gradient variations of polymers under ultraviolet radiation. Journal of Applied Polymer Science, 2007. 106(5): p. 3253-3258.

25. Hexig, B., et al., Novel biodegradable poly(butylene succinate)/poly (ethylene oxide) blend film with compositional and spherulite-size gradients. Journal of Polymer Science Part B-Polymer Physics, 2005. 43(4): p. 368-377.

26. Zhu, Y.B., et al., A new technique for preparing a filled type of polymeric gradient material. Macromolecular Materials and Engineering, 2006. 291(11): p. 1388-1396.

27. Wen, B.Y., G. Wu, and J. Yu, A flat polymeric gradient material: preparation, structure and property. Polymer, 2004. 45(10): p. 3359-3365. 
28. Behl, M., et al., One-Step Process for Creating Triple-Shape Capability of AB Polymer Networks. Advanced Functional Materials, 2009. 19(1): p. 102-108.

29. Bellin, I., S. Kelch, and A. Lendlein, Dual-shape properties of triple-shape polymer networks with crystallizable network segments and grafted side chains. Journal of Materials Chemistry, 2007. 17(28): p. 2885-2891.

30. Fischer, J. and M. Wegener, Three-dimensional optical laser lithography beyond the diffraction limit. Laser \& Photonics Reviews, 2013. 7(1): p. 22-44.

31. Luo, X.F. and P.T. Mather, Triple-Shape Polymeric Composites (TSPCS). Advanced Functional Materials, 2010. 20(16): p. 2649-2656.

32. Xie, T., X.C. Xiao, and Y.T. Cheng, Revealing Triple-Shape Memory Effect by Polymer Bilayers. Macromolecular Rapid Communications, 2009. 30(21): p. 1823-1827.

33. Bellin, I., et al., Polymeric triple-shape materials. Proceedings of the National Academy of Sciences of the United States of America, 2006. 103(48): p. 18043-18047.

34. Xie, T., Tunable polymer multi-shape memory effect. Nature, 2010. 464(7286): p. 267270.

35. Xie, T., K.A. Page, and S.A. Eastman, Strain-Based Temperature Memory Effect for Nafion and Its Molecular Origins. Advanced Functional Materials, 2011. 21(11): p. 2057-2066.

36. $\mathrm{Yu}, \mathrm{K}$., et al., Mechanisms of multi-shape memory effects and associated energy release in shape memory polymers. Soft Matter, 2012. 8(20): p. 5687-5695.

37. Li, J.J. and T. Xie, Significant Impact of Thermo-Mechanical Conditions on Polymer TripleShape Memory Effect. Macromolecules, 2011. 44(1): p. 175-180.

38. Bogue, R., 3D printing: the dawn of a new era in manufacturing? Assembly Automation, 2013. 33(4): p. 307-311.

39. Bredt, J.F., New Life for 3D Printing. R\&D Magazine, 2012. 54(2): p. 12-13.

40. Campbell, T.A. and O.S. Ivanova, 3D printing of multifunctional nanocomposites. Nano Today, 2013. 8(2): p. 119-120.

41. Hockaday, L.A., et al., Rapid 3D printing of anatomically accurate and mechanically heterogeneous aortic valve hydrogel scaffolds. Biofabrication, 2012. 4(3).

42. Martinez, E., et al., 3d Microstructural Architectures for Metal and Alloy Components Fabricated by 3d Printing/Additive Manufacturing Technologies. Proceedings of the 1st International Conference on 3d Materials Science, 2012: p. 73-78.

43. Richards, D.J., et al., 3D Printing for Tissue Engineering. Israel Journal of Chemistry, 2013. 53(9-10): p. 805-814.

44. Zhang, J.H., 3D Printing Technology-A New Mode of Computer-aided Style Design in This New Age. Industrial Instrumentation and Control Systems Ii, Pts 1-3, 2013. 336-338: p. 1383-1386.

45. Ge, Q., et al., Active Origami by 4D Printing. Smart Materials \& Structures, 2014. 23: p. 094007-15.

46. Ge, Q., H.J. Qi, and M.L. Dunn, Active materials by four-dimension printing. Applied Physics Letters, 2013. 103: p. 131901.

47. Stiltner, L.J., A. M. Elliott, and C.B. Williams, A method for creating actuated joints via fiber embedding in a polyjet $3 D$ printing process. 22nd Annual International Solid Freeform Fabrication Symposium, 2011: p. August 8-10, Austin, TX.

48. $\mathrm{Yu}, \mathrm{K}$., et al., A thermomechanical constitutive model for an epoxy based shape memory polymer and its parameter identifications. Mechanics of Time-Dependent Materials, 2014. 18(2): p. 453-474.

49. Smith, M.L., et al., Design of polarization-dependent, flexural-torsional deformation in photo responsive liquid crystalline polymer networks. Soft Matter, 2014. 10(9): p. 1400. 
50. Castro, F., et al., Effects of thermal rates on the thermomechanical behaviors of amorphous shape memory polymers. Mechanics of Time-Dependent Materials, 2010. 14(3): p. 219-241.

51. $\mathrm{Yu}, \mathrm{K} ., \mathrm{Q} . \mathrm{Ge}$, and H.J. Qi, Reduced time as a unified parameter determining fixity and free recovery of shape memory polymers. Nat Commun, 2014. 5: p. 3066.

52. Lee, J.H., et al., Highly stretchable piezoelectric-pyroelectric hybrid nanogenerator. Adv Mater, 2014. 26(5): p. 765-9.

53. Yu, K. and H.J. Qi, Temperature Memory Effect in Amorphous Shape Memory Polymers. Soft Matter, 2014. 